\title{
Uruguay gana batalla contra Philip Morris y contra el tabaquismo
}

\author{
Sergio Monraz-Pérez, Diego Sierra-Muñoz, Rogelio Pérez-Padilla $\bowtie$
}

Instituto Nacional de Enfermedades Respiratorias Ismael Cosío Villegas, Ciudad de México.

Trabajo recibido: 16-VIII-2016; aceptado: 17-VIII-2016

El tabaquismo es la principal causa prevenible de muertes en el mundo y de enfermedad respiratoria y es prioritario para los países trabajar seriamente en su reducción, para lo cual cuentan con los lineamientos del Convenio Marco de Lucha contra el Tabaquismo de la Organización Mundial de la Salud (OMS). ${ }^{1}$

En febrero del 2010 Philip Morris Internacional (PMI), la compañía tabacalera más grande del mundo con sede principal en los Estados Unidos de Norteamérica, inició una demanda contra Uruguay alegando la violación del Tratado de Inversión Bilateral (TIB) con Suiza en dos medidas de control del tabaquismo impuestas por el gobierno de Uruguay a cargo de su presidente el Dr. Tabaré Ramón Vázquez Rosas. ${ }^{2}$

La primera medida consistía en advertencias gráficas del $80 \%$ en el frente y reverso de la cajetilla; y la segunda medida fue que cada compañía tabacalera debe de limitarse a una sola variedad o marca (eliminando la idea errónea de que algunos cigarros son menos dañinos que otros) concepto conocido como Requerimiento de Presentación Única (RPU), en el Centro Internacional de Arreglo de Diferencias Relativas a Inversiones (CIADI). ${ }^{3}$

Los TIB son acuerdos realizados para proveer garantías de inversión, lo que permite a las compañías protegerse de regulaciones gubernamentales. Philip Morris alegó que el $80 \%$ de advertencia dejaba espacio insuficiente para la publicidad de sus diversas marcas, y que el Requerimiento de Presentación Única le impedía a Philip Morris comercializar algunas de sus marcas como Marlboro Dorado (Marlboro Gold), y por tanto violaba los acuerdos del TIB. Además, alegaba la falta de evidencia que demostrara la efectividad de las regulaciones propuestas, circunstancias por las cuales demandó la compensación por 25 millones de dólares.
El fallo del CIADI fue a favor del Derecho a la Salud y la Vida frente a cualquier interés comercial. Una de las decisiones más importantes fue que no es necesario demostrar una asociación directa de una regulación o medida propuesta siempre y cuando se tenga un objetivo legítimo de buena fe en protección de la salud pública.

El triunfo fue producto del esfuerzo del Programa Nacional para el Control del Tabaco del Ministerio de Salud de Uruguay, junto con un equipo multidisciplinario e interinstitucional y con el respaldo de diversas organizaciones internacionales comprometidas con la salud como el filántropo Michael Bloomberg y la fundación de Melinda y Bill Gates ${ }^{4}$ siempre en apego al Convenio Marco para el Control del Tabaco de la OMS, ${ }^{1}$ medio indispensable para la adecuada implementación de las leyes contra el tabaquismo.

De acuerdo con las principales autoridades de salud en Uruguay, el número de fumadores en este país ha disminuido desde un $35 \%$ en el 2005 , a un $22 \%$ en el 2014. ${ }^{2}$ Asimismo, el número de jóvenes fumadores cayó de $23 \%$ en el 2006, a un $8 \%$ en el 2014 , cuando Uruguay se convirtió en el primer país latinoamericano en prohibir el tabaquismo en espacios públicos cerrados. ${ }^{5}$ Otra de las medidas claves en la lucha contra el tabaquismo, que limita los sitios donde se permite fumar, reduce la aceptación social al tabaquismo y protege la salud de los que no fuman. También este país ha incrementado $15 \%$ el impuesto a los cigarrillos entre 2010 y julio 2016 , pasando del $60 \%$ al $75 \%,{ }^{6}$ estrategia que reduce de manera rápida el número de fumadores en especial jóvenes y, aún más, aumenta la recaudación. ${ }^{7}$ Aparte de los espacios libres de humo, del aumento de impuestos, de las advertencias sanitarias de gran tamaño en las cajetillas, las estrategias de salud pública contra el tabaquismo más relevantes incluyen la monitorización 
del uso del tabaco y de las políticas de prevención, la ayuda para dejar de fumar y la prohibición a las tabacaleras de publicidad y patrocinios. ${ }^{7}$

Con el resultado del litigio se reducen los riesgos de los países a demandas exitosas de la poderosa industria del tabaco por implementar medidas severas antitabaco; aunque todavía algunos se mostrarán escépticos ante la posibilidad de un litigio largo y caro, a pesar de que al final sea muy factible salir avante, siempre y cuando se tenga adecuada asesoría. ${ }^{8}$

Por muchos años, las grandes empresas productoras de bienes que afectan adversamente la salud como las del tabaco, alcohol y algunas alimentarias, han influido con su poder de diversas maneras en las decisiones de los países para tomar medidas de protección a la salud ${ }^{4}$ basadas sobre todo, pero no de forma exclusiva, en su gran poderío económico y respaldadas por los cada vez más comunes tratados comerciales internacionales.

Es pues refrescante y halagador el resultado del litigio, que esperemos se aproveche en forma adecuada por los países del mundo, y en particular América Latina para el avance en las recomendaciones del Convenio Marco de Lucha contra el Tabaquismo, con el respaldo de las Sociedades dedicadas a la atención respiratoria.

\section{REFERENCIAS}

1. Convenio Marco de la OMS para el Control del Tabaco. Organización Mundial de la Salud 2003. ISBN: 9243591010.

2. BBC Mundo. Histórico fallo a favor de Uruguay en el juicio contra la tabacalera Philip Morris Redacción. Fecha de consulta: 30/julio/2016. Disponible en: http://www. bbc.com/mundo/noticias-america-latina-36752509

3. Kaye L. Philip Morris Sues Uruguay Over Anti-Smoking Campaign. Triple Pundit 2015. Fecha de consulta: 1/ agosto/2016. Disponible en: http://www.triplepundit. com/2015/04/philip-morris-vs-uruguay-lawsuit-athreat-to-smoking-restrictions-worldwide/

4. Uruguay's Victory Over Philip Morris Will Change The World. The Huffington Post. Fecha de consulta: 21/julio/2016). Disponible en: http://www.huffingtonpost.com/laurent-huber/uruguays-victory-overphi_b_11021106.html

5. Mander B. Uruguay defeats Philip Morris test case lawsuit. Financial Times 2016. Fecha de consulta: 12/agosto/2016. Disponible en: https://next.ft.com/ content/1ae33bc8-454e-11e6-9b66-0712b3873ae1

6. Hevia M. Philip Morris vs. Uruguay: ganó la salud. Clarín. Fecha de consulta: 15/agosto/2016. Disponible en: http://www.clarin.com/opinion/Philip-Morris-Uruguaygano-salud_0_1619838115.html

7. Iniciativa libre de tabaco de la OMS. Fecha de consulta: 13/agosto/2016. Disponible en: http://www.who.int/ tobacco/mpower/publications/brochure_2013/en/

8. Kaye L. Phillip Morris loses lawsuit against Uruguay on tough tobacco rules. Triple Pundit. Fecha de consulta: 29/julio/2016. Disponible en: http://www.triplepundit. com/2016/07/uruguay-wins-lawsuit-vs-phillip-morristough-tobacco-rules/

\section{$\triangle$ Correspondencia:}

Dr. Rogelio Pérez Padilla, Instituto Nacional de Enfermedades Respiratorias Ismael Cosío Villegas. Calzada de Tlalpan Núm. 4502,Colonia Sección XVI 14080, Ciudad de México.

Correo electrónico: perezpad@gmail.com

Los autores declaran no tener conflicto de intereses. 